\title{
Two-Loop Bound-State Calculations and Squared Decay Rates
}

\author{
Ulrich D. Jentschura ${ }^{a)}$, Christoph H. Keitel ${ }^{a)}$ and Krzysztof Pachucki ${ }^{b)}$ \\ a) Fakultät Physik der Albert-Ludwigs-Universität, \\ Theoretische Quantendynamik, \\ Hermann-Herder-Straße 3, D-79104 Freiburg, Germany \\ b) Institute of Theoretical Physics, \\ University of Warsaw, ul. Hoża 69, 00-681 Warsaw, Poland
}

\begin{abstract}
We discuss the $\epsilon$-method as used in various recent QED bound-state calculations by considering mathematical model examples. Recently obtained results for higher-order self-energy binding corrections at the two-loop level are reviewed. Problems associated with the interpretation of squared decay rates as radiative bound-state energy level shifts are discussed. We briefly expand on the relation of squared decay rates to nonresonant and radiative corrections to the Lorentzian line shape, including their dependence on the experimental process under study.
\end{abstract}

PACS numbers: 31.15.-p, 12.20.Ds

Keywords: Calculations and mathematical techniques in atomic and molecular physics, quantum electrodynamics - specific calculations. 


\section{Introduction}

This paper is concerned with mathematical methods employed in recent analytic evaluations [1, 2, 3, 4, 5, 6] of higher-order binding corrections to the Lamb shift. These methods rely on a separation of the virtual photon energy integration into high- and low-energy domains. The methods are applicable in a wider context, and we attempt to provide a certain clarification by considering mathematical model examples. We focus on the two-loop self-energy correction (see Fig. 1) in hydrogenlike systems with a low nuclear charge number. We also discuss related corrections in helium.

In the second part of the paper, we discuss a recent investigation [7] which is concerned with predictive limits of energy shifts as derived from the Gell-Mann-Low-Sucher Theorem [8, 9]. Expressions obtained based on this theorem have provided the basis of level-shift calculations for decades; these may not be accurate enough for projected future experiments. Certain problems associated with this theorem find a rather natural solution in the two-time Green function method [10], other problematic aspects connected with this theorem concern the interpretation of level shifts involving squared decay rates.

Our investigations are motivated by the recent dramatic progress in laser-spectroscopic experiments in atomic hydrogen (e.g. [11,12,13]) as well as helium [14,15, 16, 17.

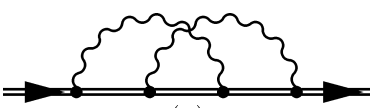

(a)

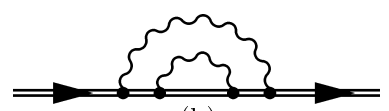

(b)

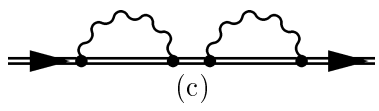

Figure 1: The crossed (a), rainbow (b) and the loop-after-loop diagram (c) which contribute to the two-loop self-energy for a bound electron. The propagator of the bound electron is denoted by a double line.

\section{The "Epsilon Method"}

In QED bound-state calculations, we are often faced with the following problem: how to separate terms of a given order in the $(Z \alpha)$-expansion, and how to treat infrared divergences that originate from higher-order terms in the expansion of the bound-electron propagator in powers of the binding field.

The so-called " $\epsilon$-method" has been employed in the analytic calculation of self-energy effects in bound systems. The energy scales for the virtual photons are treated separately: (i) the nonrelativistic domain, in which the virtual photon assumes values of the order of the atomic binding energy, and (ii) the relativistic domain, in which the virtual photon assumes values of the order of the electron rest mass. The two energy domains are separated via a parameter $\epsilon$. Without appropriate approximations and expansions applicable to the two energy domains, respectively, the analytic evaluation of either the high- or the low-energy part would not be feasible.

In one-photon calculations, we have to deal with one virtual photon energy $\omega$. For two-photon problems [4, 5, 6], one has to generalize the method to the case of two virtual quanta and, by 
consequence, two separation parameters $\epsilon_{1}$ and $\epsilon_{2}$ (see also Fig. 1 of [4]). In both the one- and the two-photon case, we require the dependence on the separation parameters to vanish at the end of the calculation, i.e. after the high- and the low-energy parts are added.

We follow here the discussion in App. A of Ref. [5], and we consider a model problem with only one "virtual photon". In contrast to Ref. [5], we choose a mathematical model problem of a slightly more complex structure in order to illustrate the occurrence of double-logarithmic terms in the semi-analytic expansion, which involves powers and logarithms of the expansion parameter. The mathematical model example reads

$$
J(\beta)=\int_{0}^{1} \ln (\omega) \sqrt{\frac{\omega^{2}+\beta^{2}}{1-\omega^{2}}} \mathrm{~d} \omega,
$$

where the integration variable $\omega$ might be interpreted as the "energy" of a "virtual photon".

We intend to derive a semi-analytic expansion of $J(\beta)$ in powers of $\beta$ and $\ln \beta$. The quantity $Z \alpha$, which parameterizes the strength of the binding Coulomb field, replaces the expansion parameter $\beta$ in actual self-energy calculations. The "high-energy part" of the calculation is given by the expression

$$
J_{\mathrm{H}}(\beta)=\int_{\epsilon}^{1} \ln (\omega) \sqrt{\frac{\omega^{2}+\beta^{2}}{1-\omega^{2}}} \mathrm{~d} \omega
$$

In the integration domain $\omega \in(\epsilon, 1)$, we may expand

$$
\sqrt{\omega^{2}+\beta^{2}}=\omega+\frac{\beta^{2}}{2 \omega}+\frac{\beta^{4}}{8 \omega^{3}}+\mathcal{O}\left(\beta^{6}\right) .
$$

However, this expansion is not applicable in higher orders to the domain $\omega \in(0, \epsilon)$ because of the appearance of inverse powers of $\omega$ which lead to "infrared divergences".

We expand the integrand of $J_{\mathrm{H}}(\beta)$ first in powers of $\beta$ according to Eq. (3). The resulting integrals can be evaluated analytically. Every term in the $\beta$-expansion is then expanded in powers of $\epsilon$ up to the order $\epsilon^{0}$. Higher-order terms in $\epsilon$ are irrelevant; they cancel at the end of the calculation (just as the divergent terms in $\epsilon$ ), because the original expression for the integral $J(\beta)$ in Eq. (11) is manifestly independent of $\epsilon$. The result of the calculation of $J_{\mathrm{H}}(\beta)$ is

$$
\begin{aligned}
& J_{\mathrm{H}}(\beta, \epsilon)=\left\{\ln (2)-1+\mathcal{O}\left(\epsilon^{2} \ln \epsilon\right)\right\}+\beta^{2}\left\{-\frac{1}{4} \ln ^{2}(\epsilon)+\frac{1}{4} \ln ^{2}(2)-\frac{\pi^{2}}{48}+\mathcal{O}\left(\epsilon^{2} \ln \epsilon\right)\right\} \\
&+\beta^{4}\left\{\frac{1}{32} \ln ^{2}(\epsilon)-\frac{1}{16 \epsilon^{2}} \ln (\epsilon)-\frac{1}{32 \epsilon^{2}}-\frac{1}{32} \ln ^{2}(2)\right. \\
&\left.+\frac{1}{32} \ln (2)+\frac{\pi^{2}}{384}+\frac{1}{64}+\mathcal{O}\left(\epsilon^{2} \ln \epsilon\right)\right\}+\mathcal{O}\left(\beta^{6} \ln ^{2} \beta\right) .
\end{aligned}
$$

The contribution $J_{\mathrm{H}}(\beta)$ corresponds to the "high-energy part" in analytic self-energy calculations, where the propagator of the bound electron may be expanded in powers of $Z \alpha$ [see Sec. III of [3]].

The expression for the low-energy part $\omega \in(0, \epsilon)$ reads

$$
J_{\mathrm{L}}(\beta)=\int_{0}^{\epsilon} \ln (\omega) \sqrt{\frac{\omega^{2}+\beta^{2}}{1-\omega^{2}}} \mathrm{~d} \omega .
$$


We have to keep the numerator of the integrand $\sqrt{\omega^{2}+\beta^{2}}$ in unexpanded form. However, within the integration domain $\omega \in(0, \epsilon)$, we may expand the denominator $\sqrt{1-\omega^{2}}$ of the integrand in powers of $\omega$. Because $\omega<\epsilon$, the expansion in powers of $\omega$ is in fact an expansion in $\beta$ in the low-energy part.

One may draw an analogy between the term $\sqrt{\omega^{2}+\beta^{2}}$ and the Schrödinger-Coulomb propagator in self-energy calculations [1, 2, 3, 5. In the low-energy domain, this propagator may not be expanded in powers of the binding field. The expansion

$$
\frac{1}{\sqrt{1-\omega^{2}}}=1+\frac{\omega^{2}}{2}+\frac{3}{8} \omega^{4}+\mathcal{O}\left(\omega^{6}\right)
$$

corresponds to the $(Z \alpha)$-expansion in the low-energy part. In actual self-energy calculations (a detailed discussion can be found in Ref. [3] ), higher-order terms in the low-energy part originate from the generalized Foldy-Wouthuysen transformation of the transition current, from relativistic corrections to the Foldy-Wouthuysen transformed Hamiltonian, and higher-order terms in the multipole expansion. Specifically, more details concerning the multipole expansion can be found in the discussion following Eq. (11) of Ref. 3.

We obtain for the low-energy part,

$$
\begin{aligned}
J_{\mathrm{L}}(\beta, \epsilon)= & \beta^{2}\left\{\frac{1}{4} \ln ^{2}(\epsilon)-\frac{1}{4} \ln ^{2}\left(\frac{2}{\beta}\right)-\frac{1}{4} \ln \left(\frac{2}{\beta}\right)-\frac{1}{8}-\frac{\pi^{2}}{24}+\mathcal{O}\left(\epsilon^{2} \ln \epsilon\right)\right\} \\
+ & \beta^{4}\left\{-\frac{1}{32} \ln ^{2}(\epsilon)+\frac{1}{16 \epsilon^{2}} \ln (\epsilon)+\frac{1}{32 \epsilon^{2}}+\frac{1}{32} \ln ^{2}\left(\frac{2}{\beta}\right)\right. \\
& \left.-\frac{1}{64} \ln \left(\frac{2}{\beta}\right)+\frac{\pi^{2}}{192}-\frac{5}{256}+\mathcal{O}\left(\epsilon^{2} \ln \epsilon\right)\right\}+\mathcal{O}\left(\beta^{6} \ln ^{2} \beta\right) .
\end{aligned}
$$

When the high-energy part (4) and the low-energy part (7) are added, the dependence on $\epsilon$ cancels, and we obtain the result

$$
\begin{aligned}
J(\beta)= & J_{\mathrm{H}}(\beta, \epsilon)+J_{\mathrm{L}}(\beta, \epsilon) \\
= & \ln (2)-1 \\
& +\beta^{2}\left\{-\frac{1}{4} \ln ^{2}\left(\frac{2}{\beta}\right)-\frac{1}{4} \ln \left(\frac{2}{\beta}\right)+\frac{1}{4} \ln ^{2}(2)-\frac{\pi^{2}}{16}-\frac{1}{8}\right\} \\
+ & \beta^{4}\left\{\frac{1}{32} \ln ^{2}\left(\frac{2}{\beta}\right)-\frac{1}{64} \ln \left(\frac{2}{\beta}\right)-\frac{1}{32} \ln ^{2}(2)+\frac{1}{32} \ln (2)\right. \\
& \left.\quad-\frac{\pi^{2}}{128}-\frac{1}{256}\right\}+\mathcal{O}\left(\beta^{6} \ln ^{2} \beta\right) .
\end{aligned}
$$

This result clearly demonstrates the semi-analytic character of the expansion: it involves doublelogarithmic terms $\ln ^{2}(2 / \beta)$ and single logarithms $\ln (2 / \beta)$ as well as constant terms. The same pattern is observed in actual self-energy calculations. 


\section{One-- and Two-Loop Self-Energy Calculations}

It is well known that the one-photon self-energy correction to the binding energy in low- $Z$ hydrogenlike systems can be parameterized as as

$$
\delta E_{\mathrm{SE}}^{(1 \gamma)}=\frac{\alpha}{\pi} \frac{(Z \alpha)^{4} m}{n^{3}} F(Z \alpha),
$$

where the dimensionless quantity $F(Z \alpha)$ has the following semi-analytic expansion,

$$
\begin{aligned}
F(Z \alpha)= & A_{41} \ln (Z \alpha)^{-2}+A_{40}+(Z \alpha) A_{50} \\
& +(Z \alpha)^{2}\left[A_{62} \ln ^{2}(Z \alpha)^{-2}+A_{61} \ln (Z \alpha)^{-2}+A_{60}+\mathcal{R}\right],
\end{aligned}
$$

where $\mathcal{R}$ vanishes as $Z \alpha \rightarrow 0$. The $A$-coefficients are state-dependent. In the following, we focus on $\mathrm{P}$ states. The coefficients $A_{41}$ and $A_{62}$ vanish for $\mathrm{P}$ states and states with higher orbital angular momenta.

In order to illustrate the analogy of our mathematical model example (see Sec. 2) with oneloop self-energy calculations, we give here the high-and low-energy parts derived in 2 for the self-energy of an electron bound in a hydrogenlike system (the $2 \mathrm{P}_{1 / 2}$ state):

$$
F_{\mathrm{H}}\left(2 \mathrm{P}_{1 / 2}\right)=-\frac{1}{6}+(Z \alpha)^{2}\left[-\frac{2}{9 \epsilon}-\frac{103}{180} \ln (\epsilon)+\frac{4177}{21600}-\frac{103}{180} \ln (2)+\mathcal{O}(\epsilon)\right]+\mathcal{O}(Z \alpha)^{3},
$$

and

$$
F_{\mathrm{L}}\left(2 \mathrm{P}_{1 / 2}\right)=-\frac{4}{3} \ln k_{0}(2 \mathrm{P})+(Z \alpha)^{2}\left[\frac{2}{9 \epsilon}+\frac{103}{180} \ln \left(\frac{\epsilon}{(Z \alpha)^{2}}\right)-0.79569(1)+\mathcal{O}(\epsilon)\right]+\mathcal{O}(Z \alpha)^{3}
$$

Adding the two contributions, the dependence on $\epsilon$ cancels, just as we had observed when forming the sum of the high-energy part (4) and the low-energy result (7).

The two-loop self-energy correction to the energy of hydrogenlike systems with low nuclear charge number reads

$$
\delta E_{\mathrm{SE}}^{(2 \gamma)}=\left(\frac{\alpha}{\pi}\right)^{2}(Z \alpha)^{4} \frac{m}{n^{3}} H(Z \alpha),
$$

where the dimensionless function $H(Z \alpha)$ is given by

$$
\begin{aligned}
H(Z \alpha)= & B_{40} \\
& +(Z \alpha)^{2}\left[B_{63} \ln ^{3}(Z \alpha)^{-2}+B_{62} \ln ^{2}(Z \alpha)^{-2}+B_{61} \ln (Z \alpha)^{-2}+B_{60}+\mathcal{R}^{\prime}\right],
\end{aligned}
$$

where $\mathcal{R}^{\prime}$ vanishes as $Z \alpha \rightarrow 0$. In two-photon calculations, we introduce two separation parameters $\epsilon_{1}$ and $\epsilon_{2}$. This leads to four different integration regions: (i) both photon energies are small (the "low-and-low-energy part"), (ii)+(iii) two mixed contributions (with one large and one small photon energy), and (iv) a "high-and-high-energy part" with two large photon energies.

$B_{63}$ vanishes for all $\mathrm{P}, \mathrm{D}, \mathrm{F}, \mathrm{G}, \ldots$ states, i.e. for all atomic states with a nonvanishing orbital angular momentum, and we first discuss here the the coefficient $B_{62}$. The low-and-low-energy part of the two-loop problem (both virtual photons have a small energy) can be evaluated using nonrelativistic quantum electrodynamics (NRQED). The relevant expression is given in 
Eq. (16) of Ref. 4]. The following double-logarithmic term of order $\alpha^{2}(Z \alpha)^{6}$ originates from the low-energy part [see Eq. (38) of Ref. [6]],

$$
\mathcal{L}=\left(\frac{\alpha}{\pi}\right)^{2} \ln \left[\frac{\epsilon_{1}}{(Z \alpha)^{2}}\right] \ln \left[\frac{\epsilon_{2}}{(Z \alpha)^{2}}\right] \frac{2 \pi\left\langle\Delta \delta^{(3)}(\boldsymbol{r})\right\rangle}{9 m^{4}},
$$

where the known result for the matrix element reads

$$
\left.\left\langle\Delta \delta^{(3)}(\boldsymbol{r})\right\rangle \equiv \Delta\left[\left|\phi_{n, l=1, m}(\boldsymbol{r})\right|^{2}\right]\right|_{r=0}=\frac{2}{3 \pi}\left[(Z \alpha)^{5} m^{5}\right] \frac{n^{2}-1}{n^{5}} .
$$

Because $B_{63}$ vanishes for $\mathrm{P}$ states, this result for the low-low-energy part determines uniquely the total result for $B_{62}$. This is because the dependence on $\epsilon_{1}$ and $\epsilon_{2}$ necessarily has to cancel at the end of the calculation according to

$$
\begin{aligned}
& \ln \left[\frac{\epsilon_{1}}{(Z \alpha)^{2} m}\right] \ln \left[\frac{\epsilon_{2}}{(Z \alpha)^{2} m}\right]+\ln \left(\frac{m}{\epsilon_{2}}\right) \ln \left[\frac{\epsilon_{1}}{(Z \alpha)^{2} m}\right] \\
& \quad+\ln \left(\frac{m}{\epsilon_{1}}\right) \ln \left[\frac{\epsilon_{2}}{(Z \alpha)^{2} m}\right]+\ln \left(\frac{m}{\epsilon_{1}}\right) \ln \left(\frac{m}{\epsilon_{2}}\right)=\ln ^{2}\left[(Z \alpha)^{-2}\right] .
\end{aligned}
$$

Note that the logarithm $\ln \left[\epsilon_{i} /(Z \alpha)^{2} m\right]$ is characteristic of the low-energy domain $(i=1,2)$, whereas the logarithm $\ln \left(m / \epsilon_{i}\right)$ is characteristic of infrared divergencies that occur in the evaluation of integrals involving highly energetic virtual photons. In view of (17), we may conclude that the coefficient of

$$
\ln \left[\frac{\epsilon_{1}}{(Z \alpha)^{2}}\right] \ln \left[\frac{\epsilon_{2}}{(Z \alpha)^{2}}\right]
$$

in the low-and-low-energy part is the same as the total coefficient of $\ln ^{2}\left[(Z \alpha)^{-2}\right]$ for the two-loop self energy. This has lead to a rigorous derivation of $B_{62}$ for $\mathrm{P}$ states [6], confirming the results of the previous investigation [18],

$$
B_{62}(n \mathrm{P})=\frac{4}{27} \frac{n^{2}-1}{n^{2}}
$$

This result is valid for all $\mathrm{P}$ states independent of the electron spin.

The $\epsilon$-method provides a convenient tool for the analysis of the problematic nonlogarithmic corrections $A_{60}$ and $B_{60}$. Let us recall that the evaluation of the one-loop coefficient $A_{60}$ for S states has attracted attention over many years $19,20,21,22,1$. Today, we can hope to evaluate the corresponding $B_{60}$-coefficients using this method.

As a first step in this direction, we have obtained results [5] for the following fine-structure differences of $B_{6 k}$-coefficients $(k=0,1)$ of $\mathrm{P}$ states,

$$
\begin{aligned}
& \Delta_{\mathrm{fs}} B_{61}=B_{61}\left(n \mathrm{P}_{3 / 2}\right)-B_{61}\left(n \mathrm{P}_{1 / 2}\right) \\
& \Delta_{\mathrm{fs}} B_{60}=B_{60}\left(n \mathrm{P}_{3 / 2}\right)-B_{60}\left(n \mathrm{P}_{1 / 2}\right) .
\end{aligned}
$$

We implicitly define the symbol $\Delta_{\mathrm{fs}}$ to denote the difference of the coefficient for an $n \mathrm{P}_{3 / 2}$ state and the corresponding $n \mathrm{P}_{1 / 2}$ level. Note that the fine-structure difference of the leading double logarithm vanishes [see Eq. (18)],

$$
\Delta_{\mathrm{fs}} B_{62}=0 .
$$

Certain simplifications are possible when considering the fine-structure difference of the $B_{61}$ and $B_{60}$-coefficients. Specifically, the contribution of the high-and-high-energy integration domain 
can be investigated with the help of a modified Dirac Hamiltonian. In this context, vertex corrections are taken into account by considering the electron form factor $F_{1}$ and $F_{2}$. A further simplification occurs because it is possible to devise a unified treatment for both the low-andlow-energy domain and the mixed-energy contributions. Some of the mixed-energy effects can be described by magnetic form-factor corrections to the leading one-photon self-energy. Because the magnetic form factor $F_{2}$ does not have infrared divergences, contributions of the type

$$
\ln \left(\frac{m}{\epsilon_{i}}\right) \ln \left[\frac{\epsilon_{3-i}}{(Z \alpha)^{2} m}\right] \quad(i=1,2)
$$

vanish for the fine-structure difference. We are therefore left with an infrared-safe and (in the context of the effective form-factor treatment) also ultraviolet-safe mixed-energy integration domain, for which a simplified treatment is possible.

We recall here the relevant results from [5]. The high-and-high-energy integration domain yields

$$
E_{\mathrm{H}}=E_{1}+E_{2 a}+E_{2 b}+E_{3},
$$

where the correction $E_{1}$ is due to the $F_{1}$ form factor, $E_{2 a}$ and $E_{2 b}$ are due to the magnetic $F_{2}$ form factor, and $E_{3}$ is caused by a second-order effect involving two one-loop magnetic form factor corrections to the spin-orbit interaction. The results read [5, Eq. (23)]

$$
E_{1}=\left(\frac{\alpha}{\pi}\right)^{2} \frac{(Z \alpha)^{6}}{n^{3}}\left[-F_{1}^{\prime(4)}(0) \frac{n^{2}-1}{n^{2}}\right] m^{3},
$$

and according to [5, Eq. (27)]

$$
E_{2 a}=\left(\frac{\alpha}{\pi}\right)^{2} \frac{(Z \alpha)^{6}}{n^{3}}\left[F_{2}^{(4)}(0)\left(\frac{487}{720}+\frac{5}{8 n}-\frac{23}{20 n^{2}}\right)\right] m
$$

as well as [5, Eq. (36)]

$$
E_{2 b}=\left(\frac{\alpha}{\pi}\right)^{2} \frac{(Z \alpha)^{6}}{n^{3}}\left[-\frac{1}{6} \frac{n^{2}-1}{n^{2}}\left(\ln \frac{m}{2 \epsilon_{1}}+\ln \frac{m}{2 \epsilon_{2}}\right)-\left(\frac{5}{18}+2 \mathcal{F}_{2}^{(4)}(0) m^{2}\right) \frac{n^{2}-1}{n^{2}}\right] m,
$$

and according to [5, Eq. (30)]

$$
E_{3}=\left(\frac{\alpha}{\pi}\right)^{2} \frac{(Z \alpha)^{6}}{n^{3}}\left[\frac{227}{2880}+\frac{1}{32 n}-\frac{3}{80 n^{2}}\right] m .
$$

Analytic results are known [23.24,25] for the two-loop form factors entering into these expressions (we take into account only the diagrams in Fig. 1 and ignore the vacuum-polarization insertion in the one-loop vertex correction),

$$
\begin{aligned}
m^{2} F_{1}^{(4)}(0) & =-\frac{47}{576}-\frac{175}{144} \zeta(2)+3 \zeta(2) \ln 2-\frac{3}{4} \zeta(3), \\
F_{2}^{(4)}(0) & =-\frac{31}{16}+\frac{5}{2} \zeta(2)-3 \zeta(2) \ln 2+\frac{3}{4} \zeta(3) \\
m^{2} \mathcal{F}_{2}^{\prime(4)}(0) & =-\frac{151}{240}+\frac{61}{40} \zeta(2)-\frac{23}{10} \zeta(2) \ln 2+\frac{23}{40} \zeta(3)
\end{aligned}
$$


The sum of the low-and-low-energy domain and the mixed integration regions is

$$
E_{\mathrm{L}}=E_{4}+E_{5}
$$

where the contribution $E_{4}$ reads [5, Eq. (51)]

$$
E_{4}=E_{4 a}+E_{4 b}=\left(\frac{\alpha}{\pi}\right)^{2} \frac{(Z \alpha)^{6} m}{n^{3}}\left[-\frac{n^{2}-1}{6 n^{2}}\left(\ln \frac{2 \epsilon_{1}}{(Z \alpha)^{2} m}+\ln \frac{2 \epsilon_{2}}{(Z \alpha)^{2} m}\right)+\frac{n^{2}-1}{n^{2}} \Delta_{\mathrm{fs}} \ell_{4}(n)\right],
$$

and the explicit results for the $\ell_{4}(n)$ are given by $[3,26$.

$$
\begin{aligned}
& \Delta_{\mathrm{fs}} \ell_{4}(2)=0.512559769(1), \\
& \Delta_{\mathrm{fs}} \ell_{4}(3)=0.513111333(1), \\
& \Delta_{\mathrm{fs}} \ell_{4}(4)=0.516095539(1), \\
& \Delta_{\mathrm{fs}} \ell_{4}(5)=0.518940860(1) .
\end{aligned}
$$

$E_{5}$ reads [5, Eq. (56)]

$$
E_{5}=\left(\frac{\alpha}{\pi}\right)^{2} \frac{(Z \alpha)^{6} m}{n^{3}}\left[\frac{n^{2}-1}{n^{2}} \Delta_{\mathrm{fs}} \ell_{5}(n)\right]
$$

where

$$
\begin{aligned}
& \Delta_{\mathrm{fs}} \ell_{5}(2)=-0.173344868(1), \\
& \Delta_{\mathrm{fs}} \ell_{5}(3)=-0.164776514(1), \\
& \Delta_{\mathrm{fs}} \ell_{5}(4)=-0.162263216(1), \\
& \Delta_{\mathrm{fs}} \ell_{5}(5)=-0.161165602(1) .
\end{aligned}
$$

Adding all contributions $E_{1}-E_{5}$, the dependence on both $\epsilon_{1}$ and $\epsilon_{2}$ cancels, and we obtain

$$
\Delta_{\mathrm{fs}} B_{61}=-\frac{n^{2}-1}{3 n^{2}}
$$

as well as

$$
\begin{aligned}
& \Delta_{\mathrm{fs}} B_{60}=\left(\frac{227}{2880}+\frac{1}{32 n}-\frac{3}{80 n^{2}}\right)+F_{2}^{(4), S}(0)\left(\frac{487}{720}+\frac{5}{8 n}-\frac{23}{20 n^{2}}\right) \\
& \quad+\frac{n^{2}-1}{n^{2}}\left[-\left(F_{1}^{\prime(4), S}(0)+2 \mathcal{F}_{2}^{\prime(4), S}(0)\right) m^{2}-\frac{5}{18}+\Delta_{\mathrm{fs}} \ell_{4}(n)+\Delta_{\mathrm{fs}} \ell_{5}(n)\right] .
\end{aligned}
$$

The explicit results for the principal quantum numbers $n=2-5$ read

$$
\begin{aligned}
& \Delta_{\mathrm{fs}} B_{60}(2)=-0.361196470(1), \\
& \Delta_{\mathrm{fs}} B_{60}(3)=-0.410149385(1), \\
& \Delta_{\mathrm{fs}} B_{60}(4)=-0.419926624(1), \\
& \Delta_{\mathrm{fs}} B_{60}(5)=-0.420872513(1) .
\end{aligned}
$$

These results have recently been generalized to the case of helium [27] and used for an estimate of higher-order binding corrections to the large and small fine-structure intervals of the triplet P levels.

At this point we would like to mention the further recent progress in the understanding of higherorder binding two-loop self-energy corrections (see Refs. [28 29 30]), which is faced with a number of conceptual and calculational difficulties, both in analytic as well as numerical approaches. 


\section{Squared Decay Rates}

An intriguing problem of bound-state quantum electrodynamics is the interpretation of squared decay rates which follow from the Gell-Mann-Low-Sucher theorem [8 9] when applied to excited atomic states in two-loop order. We have recently shown (Ref. [7]) that the squared decay rates cannot be interpreted in a natural way as real energy shifts. Roughly speaking, the problems in the interpretation originate from the fact that the Gell-Mann-Low-Sucher formalism involves a priori asymptotic states with an infinite lifetime (vanishing decay rate). The decay rate which enters in one-loop order adds an imaginary part to the energy whose square cannot be interpreted consistently as an energy shift within a formalism whose starting point was a theory that involves asymptotic states with zero decay width (for a more detailed discussion see Ref. [7]) Rather, a part of the problematic corrections can be incorporated in a natural way in a modified boundelectron Green function according to Eq. (27) of Ref. 7] which involves a "decay-rate operator" $\hat{\Gamma}$ defined in Eq. (24) of Ref. [7]. Our formula (27) of Ref. [7, which is equivalent to Eq. (16) on p. 218 of Ref. [31, could be interpreted to suggest that the modified Green function simply has a pole on the second (unphysical) sheet of the Riemann surface (see also the discussion on p. 217 of Ref. 31]). However, this is not the case: the only correction of the "squared-decay rate" type which can be incorporated in a natural way into the electron Green function formalism is the one caused by the loop-after-loop diagram in Fig. 1 (c), which is discussed in Eqs. (9) - (14) of Ref. [7. The interpretation of the other problematic corrections of the type of a "squareddecay rate" discussed in Ref. [7] [see Eqs. (5), (16), and (18) ibid.] cannot be given as easily. In order to go beyond the predictive limit of the current theory set by the squared decay, one has to consider, in a fully gauge-invariant treatment, the excitation of the atom from the ground state via the absorption of (laser) photons, and the return to the ground state via spontaneous emission. Resummations of sets of diagrams near resonance may be required. It has been stressed in Ref. [7] that the ground state is the only "true" asymptotic state which may be used as an in- and out-state in scattering theory.

We would like to stress here that the two-time Green function method [10] avoids a number of problems associated with the Gell-Mann-Low-Sucher theorem. Aside from the simplified treatment of degenerate states, we would like to mention the well-known fact that the infinitesimally damped $S_{\epsilon, \lambda}$-matrix [see Eq. (2) of Ref. [7] is, strictly speaking, not renormalizable because the damping parameter $\epsilon$ breaks the covariance.

A further problematic aspect of spectroscopic measurements is given by the nonresonant corrections [32, 33, 34, 35]. It has been stressed in 34] that nonresonant terms are enhanced in differential vs. total cross sections, and estimates for the effect in hydrogenic S-P transitions have been obtained (see Sec. 3 of [34]). The enhancement of nonresonant effects in differential as compared to total cross sections also follows in a natural way from the two-time Green function formalism [see Eqs. (3.1.120) and (3.1.121) of [10]. We observe that the experimental accuracy is approaching the $1 \mathrm{MHz}$ level at which the nonresonant terms become relevant (e.g. 36]).

The order-of-magnitude at which the nonresonant terms enter in two-photon transitions depends crucially on the process under study [34,35. After the two-photon absorption, the atom may return to the ground state via spontaneous emission of two photons. In this case, the contribution of nonresonant terms (on the level of $10^{-14} \mathrm{~Hz}$ ) is negligible at current and projected levels of experiment accuracy [34. However, this does not imply that the experimental line shape should remain Lorentzian up to this level of accuracy. For this process, the dominant correction to the Lorentzian line-shape in the two-photon transition is given by radiative (not off-resonant!) corrections, and an estimate of a relative contribution of order $\alpha(Z \alpha)^{2}$ has been given in 34 . 
(see Fig. 3 ibid., this translates into $\sim 10^{-6} \mathrm{~Hz}$ for the $1 \mathrm{~S}-2 \mathrm{~S}$ transition in atomic hydrogen). In the current experiment [13], the excited hydrogen atom $(2 \mathrm{~S})$ is quenched to the rapidly decaying $2 \mathrm{P}$ state. This leads to an experimental line width of the order of a $\mathrm{kHz}$. In this case, the nonresonant terms are enhanced, as argued in [35], and an estimate of nonresonant corrections of the order of $10^{-2} \mathrm{~Hz}$ has been given for this different experimental setup.

In all cases where off-resonant effects were considered, a formula of the general structure

$$
\frac{[\text { experimental decay width } \Gamma]^{2}}{[\text { typical atomic energy level difference } \Delta E]}
$$

has been obtained for the magnitude of the problematic shift of the peak of the photon scattering cross section (which is a nonresonant correction to the Lorentzian line shape). The meaning of the "typical atomic energy level difference" depends on the process under study: for differential cross sections in hydrogenic S-P transitions, a fine-structure level difference should be used for $\Delta E$ (see Ref. 34]), whereas for total cross sections, the correct estimate is obtained by inserting energy differences between states with a different principal quantum number (see Ref. 33]). The order-of-magnitude estimate (40) implies that (i) nonresonant effects are smaller than the experimental line width by roughly a factor of $\Gamma / \Delta E$ and (ii) the magnitude of the nonresonant terms decreases at decreasing experimental line width. A formula similar to (40) can be used in order to estimate the order-of-magnitude of the energy level "shift" by squared decay rates.

\section{Conclusions}

We have discussed the evaluation of higher-order binding corrections to the one-and two-loop self energy via the $\epsilon$-method (Sec. 2) This method has proven to be a useful calculational tool, as it leads to a rather clear separation of terms which contribute at different orders in the $(Z \alpha)$-expansion and to a transparent formulation of the physical problem. A first step in a systematic investigation of the highly problematic nonlogarithmic $B_{60}$-coefficient for hydrogenic bound states is presented. The cancellation of the expansion parameter $\epsilon$ at the end of the calculation is demonstrated by way of a mathematical model example [Eqs. (10) - (8)] and in concrete QED bound-state calculations at the one- and two-loop level (see Sec. 31).

When performing two-loop self-energy calculations for excited states, one is lead in a natural way to the problem of the interpretation of squared decay rates. These effects cannot be interpreted self-consistently as radiative energy shifts of a specific atomic energy level (Sec. 4). We consider the connection to the Gell-Mann-Low-Sucher theorem and to nonresonant corrections to the Lorentzian line shape. The process dependence of corrections to the Lorentzian line shape in twophoton transitions is analyzed, and an order-of-magnitude estimate for off-resonant corrections to the peak of the photon scattering cross section is given [see Eq. (40)].

\section{Acknowledgements}

U.D.J. and C.H.K. gratefully acknowledge funding by the Deutsche Forschungsgemeinschaft (Nachwuchsgruppe within the Sonderforschungsbereich 276), and K.P. acknowledges support from the Polish Committee for Scientific Research under contract no. 2P03B 05718. 


\section{References}

[1] K. Pachucki, Ann. Phys. (N. Y.) 226, 1 (1993).

[2] U. D. Jentschura and K. Pachucki, Phys. Rev. A 54, 1853 (1996).

[3] U. D. Jentschura, G. Soff, and P. J. Mohr, Phys. Rev. A 56, 1739 (1997).

[4] K. Pachucki, Phys. Rev. A 63, 042503 (2001).

[5] U. D. Jentschura and K. Pachucki, J. Phys. A 35, 1927 (2002).

[6] U. D. Jentschura and I. Nandori, Phys. Rev. A 66, 022114 (2002).

[7] U. D. Jentschura, J. Evers, C. H. Keitel, and K. Pachucki, New J. Phys. 4, 49 (2002).

[8] M. Gell-Mann and F. Low, Phys. Rev. 84, 350 (1951).

[9] J. Sucher, Phys. Rev. 107, 1448 (1957).

[10] V. M. Shabaev, Phys. Rep. 356, 119 (2002).

[11] B. de Beauvoir, F. Nez, L. Julien, B. Cagnac, F. Biraben, D. Touahri, L. Hilico, O. Acef, A. Clairon, and J. J. Zondy, Phys. Rev. Lett. 78, 440 (1997).

[12] C. Schwob, L. Jozefowski, B. de Beauvoir, L. Hilico, F. Nez, L. Julien, F. Biraben, O. Acef, A. Clairon, and J. J. Zondy, Phys. Rev. Lett. 82, 4960 (1999).

[13] M. Niering, R. Holzwarth, J. Reichert, P. Pokasov, T. Udem, M. Weitz, T. W. Hänsch, P. Lemonde, G. Santarelli, M. Abgrall, P. Laurent, C. Salomon, and A. Clairon, Phys. Rev. Lett. 84, 5496 (2000).

[14] E. G. Myers and M. R. Tabutt, Phys. Rev. A 61, 010501 (1999).

[15] E. G. Myers, H. S. Margolis, J. K. Thompson, M. A. Farmer, J. D. Silver, and M. R. Tabutt, Phys. Rev. Lett. 82, 4200 (1999).

[16] C. H. Storry, M. C. George, and E. A. Hessels, Phys. Rev. Lett. 84, 3274 (2000).

[17] M. C. George, L. D. Lombardi, and E. A. Hessels, Phys. Rev. Lett. 87, 173002 (2001).

[18] S. G. Karshenboim, J. Phys. B 29, L29 (1996).

[19] G. W. Erickson and D. R. Yennie, Ann. Phys. (N. Y.) 35, 271 (1965).

[20] G. W. Erickson and D. R. Yennie, Ann. Phys. (N. Y.) 35, 447 (1965).

[21] G. W. Erickson, Phys. Rev. Lett. 27, 780 (1971).

[22] J. Sapirstein, Phys. Rev. Lett. 47, 1723 (1981).

[23] E. Remiddi and J. A. M. Vermaseren, Int. J. Mod. Phys. A 15, 725 (2000).

[24] T. Gehrmann and E. Remiddi, Comput. Phys. Commun. 141, 296 (2001).

[25] P. Mastrolia and E. Remiddi, private communication (2001). 
[26] The result for the term $\Delta_{\mathrm{fs}} \ell_{4}(n=3,5)$ as given in Eq. (31) and the fine-structure difference $\Delta_{\mathrm{fs}} B_{60}(n=3,5)$ as indicated in Eqs. (37) and (39) receive small corrections as compared to results reported previously in Ref. [5] (the difference is about $1 \mathrm{~Hz}$ in frequency units for atomic hydrogen). Details of a recent evaluation of relevant relativistic higher-order binding corrections to the bound-electron self-energy are presented in E. O. LeBigot, U. D. Jentschura, P. J. Mohr, G. Soff and P. J. Indelicato, e-print physics/0304068 [for higher excited P and D states, see U. D. Jentschura, E. O. LeBigot, P. J. Mohr, P. J. Indelicato and G. Soff, Phys. Rev. Lett. 90, 163001 (2003)]. Some details of the numerical algorithms employed in this evaluation can be found in S. V. Aksenov, M. A. Savageau, U. D. Jentschura, J. Becher, G. Soff and P. J. Mohr, e-print math.NA/0207086 [Comput. Phys. Commun. 150, 1 (2003)].

[27] K. Pachucki and J. Sapirstein, J. Phys. B 35, 1783 (2002).

[28] V. A. Yerokhin, Phys. Rev. A 62, 012508 (2000).

[29] V. A. Yerokhin, Phys. Rev. Lett. 86, 1990 (2001).

[30] V. A. Yerokhin and V. M. Shabaev, Phys. Rev. A 64, 062507 (2001).

[31] C. Cohen-Tannoudji, J. Dupont-Roc, and G. Grynberg, Atom-Photon Interactions (J. Wiley \& Sons, New York, 1992).

[32] F. Low, Phys. Rev. 88, 53 (1952).

[33] L. N. Labzowsky, D. A. Solovyev, G. Plunien, and G. Soff, Phys. Rev. Lett. 87, 143003 (2001).

[34] U. D. Jentschura and P. J. Mohr, Can. J. Phys. 80, 633 (2002).

[35] L. N. Labzowsky, D. A. Solovyev, G. Plunien, and G. Soff, Phys. Rev. A 65, 054502 (2002).

[36] K. S. E. Eikema, J. Walz, and T. W. Hänsch, Phys. Rev. Lett. 86, 5679 (2001). 